\title{
Inventario de la ictiofauna de la cuenca media del río Anchicayá y selección de especies con potencial para cultivo
}

\section{Inventory of the ichthyofauna from the middle reaches of the Anchicaya River and their potential for culture}

\author{
Jorge Augusto Angulo, Biol ${ }^{*}$, Lury Nohemy García, MSc* \\ RESUMEN
}

Se realizó un inventario de peces en la cuenca media del río Anchicayá (Valle del Cauca), para actualizar la información de la ictiofauna de la zona y se hizo una preselección de las especies que pueden ser aptas para acuicultura teniendo en cuenta criterios como: su abundancia y frecuencia en las capturas, tamaño, forma, color y aceptación para el consumo por parte la comunidad local. Se capturaron 323 peces en nueve órdenes, 16 familias y 29 especies. Las especies más abundantes fueron la carduma Atherinella serrivomer (26.3\%), sabaleta Brycon henni (23.5\%), aguja Strongylura fluviatilis (11.4\%), arrayán Hemieleotris latifasciata (7.4\%) y la chuchulapa Rineloricaria jubata (6.8\%). Los órdenes con mayor número (n) de especies fueron Siluriformes (9), Perciformes (8), Characiformes (6) y Gymnotiformes (1). La comunidad seleccionó 38\% de las especies, con preferencia para consumo; $28 \%$ se puede considerar para cultivo ornamental y 31.2\% hace parte de la ictiofauna no comercial pero puede poseer gran importancia ecológica, como Atherinella serrivomer, que por su abundancia, se valora como indicador biológico de la zona. Las capturas señalan que existen un buen porcentaje de peces nativos con características apropiadas para el desarrollo de cultivo.

Palabras clave: Acuicultura; Ictiofauna; Inventarios; Peces nativos y talasoides; Punto caliente de biodiversidad.

\begin{abstract}
An inventory of fish was realised in the average river basin of the Anchicaya River (Valle del Cauca), to update the information of ictiofauna of the zone and realized a preselection of the species that can be apt for aquiculture considering criteria like: its abundance and frequency in the captures, size, form color and acceptance for the consumption by part the local community. 323 fish in 9 orders, 16 families and 29 species captured themselves. The most abundant species were carduma Atherinella to serrivomer (26.3\%), sabaleta Brycon henni (23.5\%), needle Strongylura fluviatilis (11.4\%), arrayán latifasciata Hemieleotris (7.4\%) and chuchulapa Rineloricaria jubata (6.8\%). You order them with greater number (n) of species were Siluriformes (9), Perciformes (8), Characiformes (6) and Gymnotiformes (1). The community I select $38 \%$ of the species, with preference for consumption; $28 \%$ can be considered for ornamental culture and $31.2 \%$ are part of ictiofauna noncommercial but they can own great ecological importance, like Atherinella serrivomer, that by its abundance, has to be valued like biological indicador of the zone. The captures indicate that a good percentage of native fish with appropriate typical for the development of culture exists.
\end{abstract}

* Docente, Programa Tecnología en Acuicultura, Universidad del Pacífico, Buenaventura, Colombia. e-mail:joraugus@yahoo.com acuicultura@unipacifico.edu.co

Recibido: 24 de junio, 2010 Aceptado: 18 de agosto, 2010 
Keywords: Aquaculture; Biological hotspots; Fish inventory; Ichthyofauna; Native fishes; Thallasoid fishes.

\section{INTRODUCCIÓN}

Uno de los recursos con alto potencial de aprovechamiento en la costa Pacífica colombiana son los peces nativos de aguas dulces, pero su cultivo requiere de amplio conocimiento del comportamiento biológico y ecológico de estos organismos en su medio natural. En la actualidad, en el Centro de Investigación y Producción Acuícola Henry von Phral de la Universidad del Pacífico, Buenaventura, se realizan investigaciones en este sentido, en busca de especies nativas aptas para el cultivo, que contribuyan a mejorar la calidad de vida del poblador rural del Pacífico colombiano. A nivel global, la importancia del río Anchicayá es que se encuentra en la zona biogeográfica de Tumbes-Chocó-Magdalena. Esta zona es una de las regiones más ricas en diversidad biológica del planeta pero al mismo tiempo amenazadas con alto impacto de la actividad humana. La zona es conocida o designada «a biological hotspot o punto caliente de biodiversidad» por instituciones internacionales para la protección del medio ambiente (http://www.biodiversityhotspots.org/xp/hotspots/ tumbes_choco/Pages/default.aspx).

Son varios los autores que han estudiado la ictiofauna del río Anchicayá. El primer informe para este río fue realizado por Miles en 1943 (publicado hasta el año 1971), que en su estudio de los peces del Valle del Cauca registró nueve especies provenientes de los ríos Dagua y Anchicayá. Ospina y Restrepo (1989) en su trabajo de grado realizado en el río Sabaletas (afluente del Anchicayá) presentaron 33 especies de peces. Los trabajos recientes de Tovar (2004) y Tovar et al. (2007) informaron 45, 35, y 21 especies respectivamente para las zonas alta, media y baja del río.

Si bien con este trabajo se busca sobre todo realizar un muestreo para formular un inventario y actualizar la información de la ictiofauna de la cuenca media del río Anchicayá, se complementa con una primera selección de especies de peces nativos de la cuenca que cumplen con criterios para el cultivo de consumo o como ornamental.

\section{MATERIALES Y MÉTODOS}

Area de estudio. La cuenca hidrográfica del río Anchicayá se localiza en la vertiente del Pacífico de la cordillera Occidental, en la jurisdicción de los municipios de Buenaventura y Dagua, el Parque Nacional Natural Farallones de Cali, donde nace el río, hasta la desembocadura en la bahía de Buenaventura. Tiene una superficie de 130.568 hectáreas, distribuidas en los municipios de Buenaventura y Dagua (Quiroga-Zea et al. 1998). Cuenta con dos embalses, bajo y alto Anchicayá, entre los kilómetros 80 y 90 de la carretera Simón Bolívar, antigua vía Buenaventura-Cali. La pluviosidad es constante (precipitaciones hasta $183 \mathrm{~mm}$ en 24 horas, en mayor proporción nocturnas), una temperatura ambiente de $26^{\circ} \mathrm{C}$, y una humedad relativa de $86 \%$. Casi todo el recorrido se realiza por una zona montañosa, recubierta por extensa zona de bosque muy húmedo tropical (Bmh-T), numerosas quebradas y riachuelos aportan gran volumen de agua a su caudal (Tovar 2004).

La zona de estudio correspondió a la zona del río Anchicayá (seis estaciones de muestreo) entre las veredas de Guaimia y Sabaletas e incluye el río Sabaletas, antes de su desembocadura y la quebrada Las Vacas (Figura 1). El área corresponde a la cuenca media del río Anchicayá según la zonificación geomorfológica realizada por Barbosa (1998; en Tovar 2004).

Captura de peces. Entre agosto de 2006 y febrero de 2008 se realizaron 15 visitas al área de estudio con el fin de realizar faenas de pesca. El trabajo de campo se realizó con el apoyo de estudiantes de la Universidad del Pacífico e integrantes del Consejo Comunitario de la vereda de Limones (Figura 2).

Se emplearon tres artes de pesca que fueron el chinchorro, trasmallo y atarraya. La técnica de pesca que se empleó con los artes fue similar realizándose en barridos sobre un área aproximada de $50 \mathrm{~m}$ a $100 \mathrm{~m}$, con el fin de capturar los peces que se encontraban en la columna de agua y aquellos de hábitos bentónicos. La duración de las pescas varió entre una y tres horas; el desplazamiento entre las estaciones se realizó en bote con motor fuera de borda. Se utilizó el chinchorro en mayor proporción (90\%), con una longitud de 20 my una altura mínima de $2 \mathrm{~m}$. Su tamaño permitió abarcar un amplio sector del río y su diámetro de malla de media pulgada, permitió colectar un amplio espectro de especies y tallas; el material de la red era polietileno. El trasmallo se usó en un 5\% y fue importante cuando el número de personas participantes en las pescas era reducido; estaba fabricado en nylon monofilamento con ojo de malla de dos pulgadas. La atarraya se empleó en un 5\% y haciendo arrastres muy cerca de la orilla; ésta tenía ojo de malla de una pulgada y radio de dos metros, fabricada en nylon multifilamento.

Identificación de especies. La identificación taxonómica de los peces se realizó mediante la revisión de claves y referencias especializadas deEigenmann(1922), Dahl(1971), Miles (1971), Castillo y Rubio (1987), Rubio (1988), Ospina y Restrepo (1989), Galvis et al. (1997) y Reis et al. (2003). Algunos ejemplares de peces capturados se fijaron en solución de formol al $10 \%$, los demás se devolvieron vivos al agua. Posteriormente, los peces fijados se preservaron en alcohol al $70 \%$. Con el material preservado se revisó la identificación de los especímenes y se hizo una colección de referencia en la Universidad del Pacífico (CRUP).

Criterio de selección de las especies. La preselección de las especies aptas para acuicultura, se hizo teniendo cuenta criterios como: abundancia, frecuencia en las capturas y su 




Figura 1. Área de estudio, cuenca media del río Anchicayá. Fuente: Barbosa 1998.

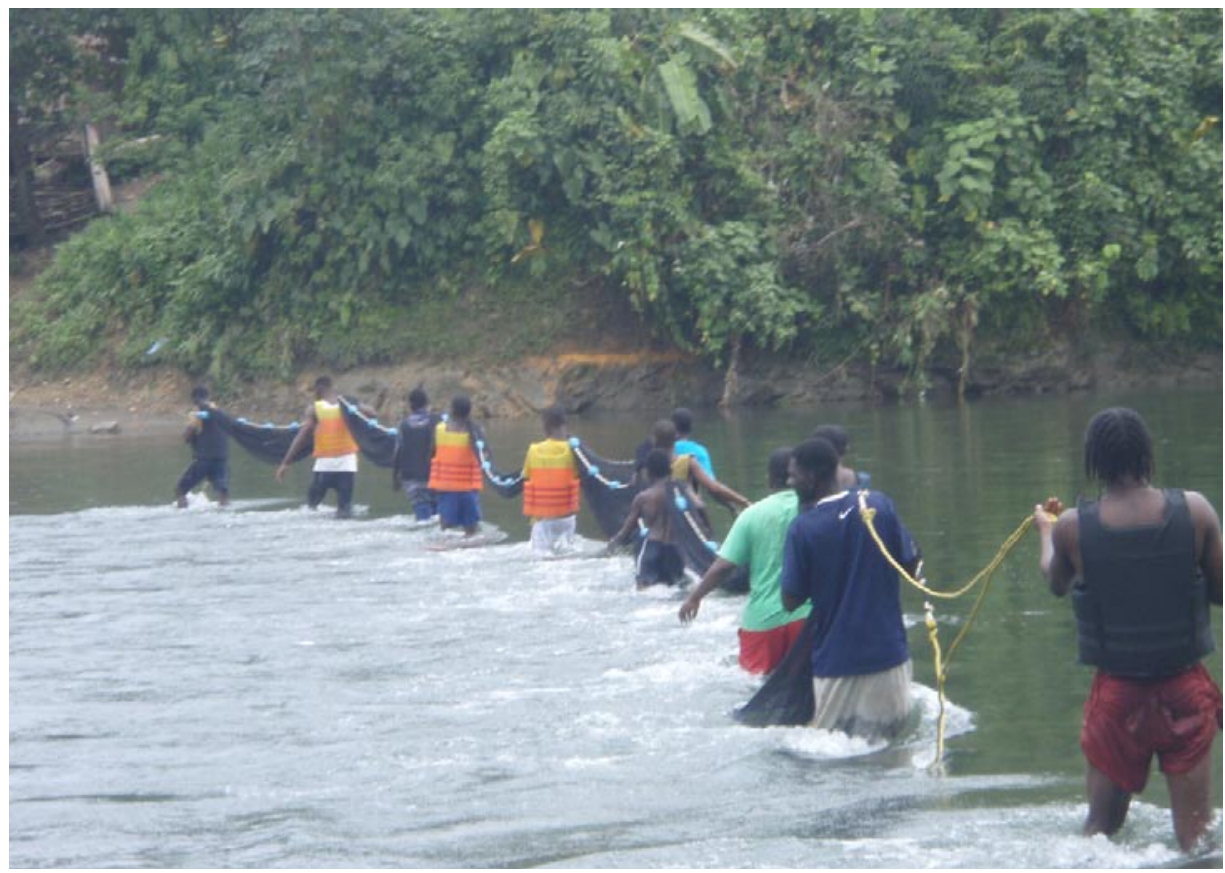

Figura 2. Faena de pesca en río Anchicayá, Valle del Cauca. Colaboración entre habitantes de las comunidades locales y estudiantes con profesorado de la Universidad del Pacífico, Buenaventura, Colombia. aceptación por la comunidad para escoger aquellas aptas para consumo; también se consideró el tamaño, forma y color para seleccionar especies con potencial ornamental. Los datos de la captura se complementaron con información generada de una encuesta a 20 habitantes de la comunidad de Sabaletas y Limones con el fin de de caracterizar las especies de peces de acuerdo con el aprovechamiento que hacen de ellas.

\section{RESULTADOS}

En las seis estaciones se capturaron y se identificaron 323 individuos distribuidos en nueve órdenes, 16 familias y 29 especies (Tabla 1). El orden con mayor número de especies fue Siluriformes con 10, Perciformes con ocho y Characiformes con seis. Los Gymnotiformes sólo estuvieron representados por un ejemplar de viringo Sternopygus sp. (Tabla 1). La familia con el mayor número de especies fue Characidae con siete que fue la única representante del orden Characiformes; le siguieron Loricaridae y Pimelodidae, con cuatro especies cada una. La familia Characidae también fue la de mayor abundancia numérica con 113 individuos (34\%). Las cinco especies más abundantes fueron en su orden, la carduma Atherinella serrivomer $(26.3 \%)$, la sabaleta Brycon henni (23.5\%); la aguja Strongylurafluviatilis (11.5\%); el arrayán Hemieleotris latifasciata $(7.4 \%)$ y la chuchulapa Rineloricariajubata (6.8\%). Las especies con mayor número de capturas fueron la carduma $A$. serrivomer y la aguja $S$. fluviatilis que son especies de origen marino. 
Ictiofauna del río Anchicayá. JA Angulo, LN García

Tabla 1

Listado total y tipo de hábitat de especies capturadas (agosto 2006-febrero 2008). Cuenca media del río Anchicayá, Valle del Cauca, Colombia

\begin{tabular}{|c|c|c|c|c|c|}
\hline & Nombre común & Nombre científico & $\mathbf{N}^{\circ}$ individuos & $\%$ & Origen \\
\hline 1 & Aguja & Strongylura fluviatilis & 37 & 11,5 & Marino \\
\hline 2 & Anguilla & Ophichthus sp. & 1 & 0,3 & Marino \\
\hline 3 & Arrayán & Hemieleotris latifasciata & 24 & 7,4 & Agua dulce \\
\hline 4 & Barbudo & Ramdia wagneri & 2 & 0,6 & Agua dulce \\
\hline 5 & Bocón & Eleotris picta & 2 & 0,6 & Agua dulce \\
\hline 6 & Bocón & Gobiomorus maculatus & 3 & 0,9 & Agua dulce \\
\hline 7 & Jabón & Cetopsis amphiloxa & 1 & 0,3 & Agua dulce \\
\hline 8 & Capitán & Cephalosilurus zungaro & 1 & 0,3 & Agua dulce \\
\hline 9 & Carduma & Atherinella serrivomer & 85 & 26,3 & Marino \\
\hline 10 & Chuchulapa & Rineloricaria jubata & 22 & 6,8 & Agua dulce \\
\hline 11 & Chuchulapa & Loricariasp. & 8 & 2,9 & Agua dulce \\
\hline 12 & Corroncho & Lasiancistrus sp. & 2 & 0,6 & Agua dulce \\
\hline 13 & Guacuco & Bunocephalus sp. & 1 & 0,3 & Agua dulce \\
\hline 14 & Guacuco & Chaetostoma sp. & 4 & 1,2 & Agua dulce \\
\hline 15 & Jojorro & Pomadasys Bayanus & 1 & 0,3 & Marino \\
\hline 16 & Jorobado & Romboides hildebrandi & 12 & 3,7 & Agua dulce \\
\hline 17 & Lambearena & Awous transandeanus & 1 & 0,3 & Agua dulce \\
\hline 18 & Lenguado & Trinectes fluviatilis & 1 & 0,3 & Marino \\
\hline 19 & Mojarra paridera & Cichlassoma atromaculatum & 3 & 0,9 & Agua dulce \\
\hline 20 & Mojarra & Caquetaia krausii & 1 & 0,3 & Agua dulce \\
\hline 21 & Nayo de pozo & Pseudocurimata lineopuntacta & 9 & 2,8 & Agua dulce \\
\hline 22 & Nayo de río & Agonostomus monticula & 2 & 0,6 & Marino \\
\hline 23 & Nicuro & Pimelodella grisea & 6 & 1,9 & Agua dulce \\
\hline 24 & Pejesapo & Daector dovi & 1 & 0,3 & Marino \\
\hline 25 & Sabaleta & Brycon henni & 76 & 23,5 & Agua dulce \\
\hline 26 & Sábalo & Brycon meeki & 1 & 0,3 & Agua dulce \\
\hline 27 & Sardina & Bryconamericus scopiferus & 11 & 3,4 & Agua dulce \\
\hline 28 & Sardina & Bryconamericus sp. & 4 & 1,2 & Agua dulce \\
\hline \multirow[t]{2}{*}{29} & Viringo & Sternopygus sp. & 1 & 0,3 & Agua dulce \\
\hline & Total & & 323 & 100.0 & \\
\hline
\end{tabular}

El 76\% de las especies capturadas se consideran peces primarios, que habitan estrictamente aguas dulces (Tabla 1). El restante $(24 \%)$ de los peces capturados son especies secundarias (o Talasoide), que tienen mayor tolerancia a la salinidad y remontan distancias considerables en este río, desde zonas estuarinas (Tabla 1). Tal es el caso del jojorro Pomadasys bayanus que puede habitar ambientes con salinidades entre 20-30 ppm (Robertson y Allen 2002). También, el pejesapo Daector dowi, habitante común de los estuarios del Pacífico centro-oriental tropical, se encontró cerca de la estación de Limones; según pescadores de la comunidad es frecuente encontrarlos en la orilla del río principal. Son también habitantes de zonas estuarinas como la bahía de Buenaventura, el bobo Eleoctris picta, el lenguado Trinectesfluviatilis, el lambearena Awous transandeanus, la lisa Chaenomugil proboscideus, el nayo de río Agonostomus monticula y la carduma Atherinella sp (Rubio 1988; Robertson y Allen 2002). La aguja S. fluviatilis es una especie de agua dulce que migra también a zonas de manglar. 


\section{Bioetnia Volumen 7 No 2 (julio-diciembre), 2010}

\section{DISCUSIÓN}

El número de especies encontradas es similar al que se halló en otros trabajos realizados en la zona con diferentes artes de pesca tales como, Ospina y Restrepo (1989) quienes identificaron 33 especies, y Tovar y Acevedo (2007) con 35 especies. Esta cantidad es relativamente baja en comparación con los informes para otras cuencas en diferentes regiones de Colombia como la Amazonía (Salinas y Agudelo 2000) y la Andina (Maldonado-Ocampo et al. 2005). Se observa también un bajo número de individuos en las capturas.

En términos generales se considera que el potencial del recurso íctico de los ríos que drenan sobre la costa pacífica es bajo, aunque con gran número de especies nativas, sobre todo por su origen reciente, por sus pendientes pronunciadas, por la longitud corta de su recorrido y por la conformación de tipo de suelos sobre los que drenan sus aguas. En el caso del río Anchicayá, se le suma la presencia de dos represas que causan un fuerte impacto sobre el río, razón por la que es lógico categorizar la zona de alta diversidad ecológica pero susceptible a degrado ambiental (punto caliente de biodiversidad). Trabajos recientes, como los de Salgado y Paz (1995) Quiroga et al. (1998), y Ramos y Ríos (1998) constituyen importantes puntos de referencia para comparar el impacto causado a la ictiofauna de la cuenca por el vertimiento de lodos desde la represa del Bajo Anchicayá en el año 2001, efecto que analizaron después INCOL y EPSA (2003). Estos datos en conjunto, confirman que la cuenca del río Anchicayá se puede ser considerar como «punto caliente de biodiversidad» dentro la zona biogeográfica de Tumbes, Chocó. Adicionalmente, frente a las condiciones actuales del río se debe considerar esta zona no apta para pesca comercial.

Para considerar el desarrollo de cultivo en cautiverio de peces nativos de la zona, fue preciso tener en cuenta criterios, como su aceptación por el consumidor y abundancia en el medio que permita obtener un plantel inicial de reproductores. Con base en la encuesta que se realizó a los pescadores de la comunidad, 38\% de las especies capturadas, se emplean para consumo; según orden de abundancia relativa en las capturas, éstas fueron la sabaleta Brycon henni (23.5\%), la aguja Strongylura fluviatilis (11.5\%), el nayo de pozo Pseudocurimata lineopuntacta (2.8\%), el nicuro Pimelodella grisea (1.9\%), las mojarras Cichlassoma atromaculatum (1\%) y Caquetaia krausii (0.3\%), el barbudo Ramdia wagneri (0.6\%) y el nayo de río Agonostomus monticula (0.3\%).

En la Universidad del Pacífico ya se realizan investigaciones con el barbudo $R$. wagneri por su valor comestible y fácil adaptación al cautiverio. Investigadores de varias instituciones nacionales también han desarrollado técnicas de cultivo para especies de bagres, bocachico, sabaleta y tilapias (Atencio-García 2001), que son aptas y se pueden transferir con facilidad a las especies nativas encontradas en la región porque pertenecen al mismo grupo taxonómico.

E1 28\% de los individuos capturados se pueden considerar como peces ornamentales como el capitán Cephalosilurus zungaro, el corroncho Chaetostoma sp., las chuchulapas Loricaria sp. y Rineloricaria cubata, los guacucos Bunocephalus sp., y Lasiancistrus sp., el arrayán Hemieleotris latifasciata, el viringo Sternopygus sp., la doncella Cetopsis amphiloxa y el lenguado Trinectes fluviatilis. Estos peces pertenecen a grupos taxonómicos ya establecidos en el mercado mundial de peces ornamentales, donde se prefieren ya sea para armar un verdadero ecosistema en el acuario comunitario como «limpiadores» biológicos de desperdicios en el caso de los bagres (podrían ser por ejemplo el capitán, corronchos, chuchulapas) o como peces ornamentales por su gran variedad de formas, bellos colores (ejemplo el arrayán), comportamientos apasionantes y adaptaciones únicas o interesantes como sensores eléctricos de comunicación, para caza o reproducción (ejemplo el viringo). Estas especies con ciclos de vida cortos y fácil mantenimiento en acuarios, permiten a nivel casero, una rápida alternativa para la identificación de mutaciones y desarrollo de programas de selección y cruzamiento para el mejoramiento genético de formas y colores deseados por la comunidad acuarista, apasionada por peces multiformes y multicolores. Ya son de amplio reconocimiento en el mercado de la acuariofilía mundial el tetra emperador Nematobrycon palmeri y el tiburoncito Arius jordani, ambas especies endémicas de la región. El tiburoncito todavía se captura de manera directa del medio ambiente mientras el emperador tetra se cultiva de forma masiva en el Asia. Estos dos ejemplos sirven para destacar la gran importancia que pueden tener los recursos ícticos de esta región y la necesidad de hacer inventarios biológicos como los que se presentan en este estudio. El resto de las especies colectadas hacen parte de la comunidad íctica del río que carecen de importancia económica, pero poseen una notable importancia ecológica como eslabón trófico para peces consumidores superiores; tal es el caso de la carduma $A$. serrivomer que resultó ser la especie más abundante de la cuenca media del río Anchicayá y ha de ser valorada como indicador biológico de la zona (Figura 3).

Si bien se puede señalar que existe un buen porcentaje de peces nativos con potencial para el desarrollo de su cultivo como pez de consumo o pez ornamental, es importante enfocar estudios en el conocimiento básico de su biología y ecología. Realizar investigaciones sobre las especies que cumplan con otros criterios tales como rápido crecimiento, reproducción en condiciones de cultivo, tolerancia en altas densidades, rusticidad o resistencia, para seleccionar las más aptas para el cultivo. 


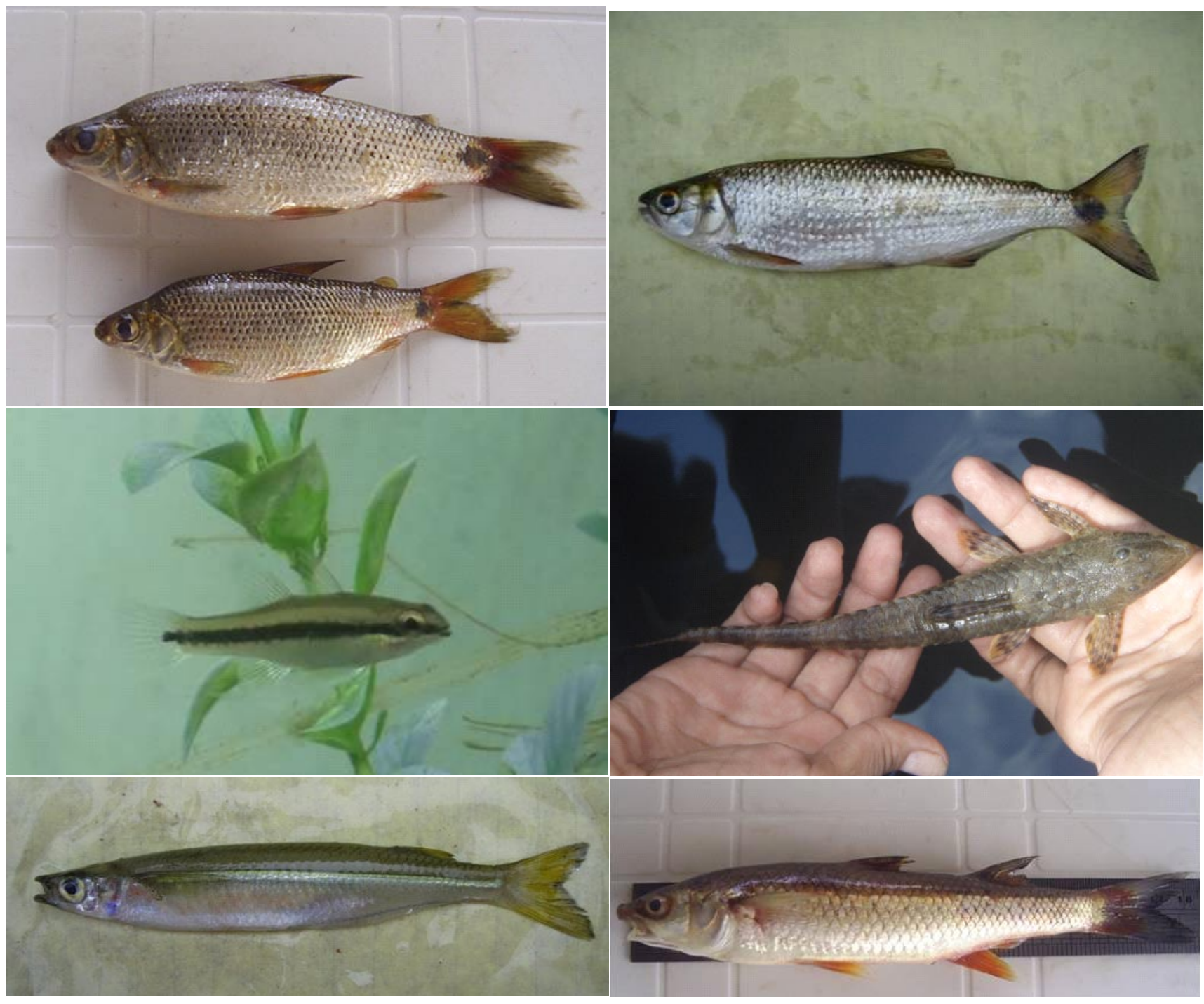

Figura 3. Peces nativos de la cuenca del río Anchicayá, Valle del Cauca, Colombia. Peces para consumo humano (Nayo de pozo Pseudocurimata lineopuntactay la sabaleta Brycon henni); peces con potencial ornamental (Arayan Hemieleotris latisfasciatay chuchulapa Rineloricaria jubata); peces con notable importancia ecológica y que se pueden utilizar para elaborar un índice de integridad biológica (Carduma Atherinella serrivomery Nayo de río Agonostomus monticula).

\section{CONCLUSIONES}

- Elnúmero de especies en la cuenca media del río Anchicayá es baja; estos resultados son similares a los citados en otros trabajos para la zona. No obstante, la variedad de especies nativas colectado, es una oportunidad única a nivel científico para el estudio de la biodiversidad íctica de los ríos de esta región. Frente a las condiciones de perturbación ecológica que se informan en la zona, el río se debe considerar sin ningún potencial pesquero comercial y asimismo, como «punto caliente de biodiversidad» dentro de la zona biogeográfica de Tumbes, Chocó. La carduma
Atherinella serrivomer, se podría valorar como indicador biológico de la zona.

- Un alto porcentaje de las especies presentes (38\%) tienen un valor directo y sirven de sustento alimenticio para las poblaciones locales. A nivel taxonómico, las características biológicas de estas especies y tecnologías de cultivo existentes en especies similares (ejemplo bagres, bocachico y ciclidos como son las tilapias) podrían ser adaptadas de manera rápida para aplicarlas en el desarrollo sostenible de las especies locales.

- Un porcentaje significativo (28\%) de las especies que se encontraron pueden tener alto valor comercial como espe- 


\section{Bioetnia Volumen 7 No 2 (julio-diciembre), 2010}

cies ornamentales. Las especies identificadas con potencial ornamental fueron el capitán Cephalosilurus zungaro, el corroncho Chaetostoma sp., las chuchulapas Loricaria sp. y Rineloricaria cubata, los guacucos Bunocephalus sp., y Lasiancistrus sp., el arrayán Hemieleotris latifasciata, el viringo Sternopygus sp., la doncella Cetopsis amphiloxa y el lenguado Trinectes fluviatilis.

\section{AGRADECIMIENTOS}

Los autores de este trabajo y la Universidad del Pacífico expresan su agradecimiento a los jóvenes integrantes del consejo comunitario de la vereda de Limones, a los estudiantes del programa de Acuicultura de la Universidad del Pacífico por su apoyo incondicional en las faenas de pesca; a la bióloga Sandra Lamoroux por la fotografía del Nayo de Pozo; al biólogo Gilbert Acevedo por información bibliográfica; al biólogo Armando Ortega por la revisión del manuscrito y a todos aquellos que hicieron posible esta publicación.

\section{LITERATURACITADA}

Atencio, V. 2001. Producción de alevinos de especies nativas. MVZ 6 (1): 9 14.

Castillo, L. F., E. A. Rubio. 1987. Estudio de la ictiofauna de los esteros y partes bajas de los ríos San Juan, Dagua y Calima, Departamento del Valle del Cauca. Cespedecia. 15-16 (53-54): 55-56.

Dahl, G. 1971. Los peces del norte de Colombia. Bogotá, DC: INDERENA; $391 \mathrm{pp}$.

Eigenmann, C. H. 1922. The fishes of western South America. Part I. The freshwater fishes of northwestern South America, including Colombia, Panama and the Pacific slopes of Ecuador and Peru, together with an Appendix upon the fishes of the Meta River in Colombia. Memories of the Carnegie Museum. 9 (1): 1-346.

Galvis, G., J. I. Mojica, M. Camargo. 1997. Peces del Catatumbo. Bogotá, DC: Asociación Cravo Norte; $188 \mathrm{pp}$.

INCOL S.A., EPSA S. A. 2003. Estudio hidrobiológico comparativo (monitoreo) en el río Anchicayá de nueve puntos adicionales. Informe
Final. Cali: EPSA; 156 pp.

Maldonado-Ocampo, J., A. Ortega-Lara, J. S. Usma, G. Galvis, F. A. VillaNavarro, L. Vásquez, et al.2005. Peces de los Andes de Colombia. Bogotá, DC: Instituto de Investigación de Recursos Biológicos «Alexander Von Humboldt»; 346 pp.

Miles, C. 1971. Los peces del río Magdalena. Bogotá, DC: Ministerio de la Economía Nacional, Sección de Piscicultura, Pesca y Caza; 106 pp.

Ospina, C. E., C. A. Restrepo. 1989. Contribución al conocimiento de la biología de peces del Bajo Anchicayá. Tesis de Grado. Palmira: Facultad de Ciencias Agropecuarias, Universidad Nacional; 162 pp.

Quiroga Zea, F., V. A. Vásquez, H. Prada, V. Cabezas Gutiérrez, J. P. MurielRojas. 1998. Plan integral de ordenamiento y manejo sostenible con participación comunitaria de la cuenca hidrográfica del río Anchicayá. Departamento del Valle del Cauca, municipios de Buenaventura y Dagua. Cali: Corporación Autónoma Regional del Valle del Cauca (CVC) y la Universidad del Valle; $128 \mathrm{pp}$.

Ramos, G., R. Ríos. 1998. Anexo 2.3. Hidrobiología, proyectos de acuicultura y vertebrados terrestres. Plan Integral de Ordenamiento y Manejo Sostenible con Participación Comunitaria para la Cuenca Hidrográfica del Río Anchicayá. Cali: Universidad del Valle, CVC; 25 pp.

Reis R. E., S. O. Kullander, C. J. Ferraris. 2003. Check list of the freshwater fishes of South and Central America. Porto Alegre: EDIPUCRS; p. 33050.

Robertson, D. R., G. R. Allen. (CD-ROM). 2002. Shore fishes of the tropical eastern Pacific. An information system. Balboa: Smithsonian Tropical Research Institute.

Rubio, E. A., 1988. Estudio taxonómico de la ictiofauna acompañante del camarón en áreas costeras del Pacífico de Colombia. Memorias del VI Seminario Nacional de las Ciencias del Mar, Comisión Colombiana de Oceanografía, Bogotá, DC; p. 169-83.

Salgado, M. M., L. B. Paz. 1995. Memorando de factibilidad $\mathrm{N}^{\circ}$ 37. Proyecto desviación del río Dagua al embalse Alto Anchicayá, organismos bentónicos como elemento de análisis en el impacto generado por la desviación del río Dagua. $\mathrm{N}^{\circ} 38$. Caracterización de la fauna íctica. Cali: EPSA; $89 \mathrm{p}$.

Salinas, Y., E. Agudelo. 2000. Peces de importancia económica en la cuenca amazónica colombiana. Bogotá, DC: Instituto Amazónico de Investigaciones Científicas, Ministerio del Medio Ambiente; 140 pp.

Tovar, J. A. 2004. Estudio hidrobiológico comparativo (monitoreo), para observar el estado actual del río Anchicayá, aguas arriba y aguas abajo de la represa del Bajo Anchicayá. Cali: EPSA; 58 pp.

Tovar, J. A., G. Acevedo. 2007. Monitoreo bimestral hidrológico y biológico en el sector aguas abajo de la presa del Bajo Anchicayá como base de un plan de repoblamiento piscicola con participación comunitaria. Informe técnico. Cali: EPSA; 72 pp. 